Its base was injected. A large artery coursed over the sclera to break up into capillaries upon the base of the tumour. There was little doubt from the appearance of the tumour that it was malignant. Transillumination gave a dark shadow in the region behind the visible growth. A diagnosis of sarcoma of the ciliary body was made and the eye was enucleated.

The eye was examined by Mr. Assinder, pathologist to the Birmingham Fye Hospital, who reported as follows :-

Endothelioma in the region of the ciliary body is of very rare occurrence. Tumours difficult of classification have often been wrongly called endotheliomata, and it is surprising how often, in some laboratories, such a diagnosis is made. I am not prepared to state definitely the exact position at which this growth originated, but it probably arose in the lymphatics in the region of the ciliary body, near the angle, and invaded the iris.

The growth itself is a non-pigmented one, although pigment from the posterior surface of the iris has been incorporated in part of the tumour.

It is composed of large flattened cells, with generally an illdefined outline, showing numerous vacuoles and comparatively large well-stained nuclei. The newly-formed vascular spaces are everywhere apparent.

According to Kettle and Ross (Lancet, May 19, 1921), one of the characteristic features, constantly seen, is the development of the intra-cellular vacuoles, and it is from these vacuoles, increasing in size and running together, that the irregular vascular spaces are formed.

It is probable that the tumour had been growing for some years. The patient had noted the loss of sight for five years. The slight iritis and the cataract were obviously due to the growth, for the left eye showed no trace of iritis or of cataract.

No pain was noted and the tension was never raised above the normal.

\title{
PROFESSOR VOGT'S COURSE ON SLIT-LAMP MICROSCOPY
}

\author{
BY \\ T. HARRISON BUTLER \\ BIRMINGHAM
}

ON September 3, 1923, Professor Vogt began his second course on Slit-Lamp Microscopy at the University Eye Clinic at Zürich. The first was held at Basle in September, 1922.

The course extended over a whole week, and was divided into two sections. Every day a lecture was given in the well-appointed 
lecture theatre from 10 to 12 , and this was followed up by practical work upon the subject of the lecture from 3 to 6 in the large dark room. This room was capacious enough to accommodate fourteen slit-lamps and forty-seven students without undue overcrowding. There were fourteen instructors : the professor himself; his four assistants; and his former assistants at Basle gathered from the various clinics of Switzerland. Dr. W. G. M. Byers, of McGill University, who had been working in the clinic, acted as one of the instructors and translated parts of the lectures into English. 450 patients had been written for.

Monday's lecture dealt with the principles of the Gullstrand slit-lamp, with its adjustments, and with the importance of being able to vary the width of the pencil of light. This pencil, in German Büschel, is really a ribbon of light, and will in future be called the "ribbon." Finally, the lecturer described the prismatic form of the illuminated area in the cornea, and impressed upon his class the vital necessity of learning to focus it sharply with one hand on the focussing screw of the illuminating lens c.f the slit-lamp, the other on the focussing screw of the microscope. Upon the clear view of the prism and the recognition of its chief sides and angles depend the power of localizing the exact position of abnormalities in the cornea, and variations in its thickness. Professor Vogt's chief assistant told us that if we had not learned to localize we had missed the whole point of the course.

Monday afternoon was devoted to the adjustment of the slitlamp, and to learning to focus the ribbon of light and the microscope at the same time. Pigs' eves were used for this practice. Attention was directed to localizing in the cornea with the ordinary ribbon.

Most of the students went home feeling that they were faced with an instrument as difficult as if not more difficult than the ophthalmoscope and the retinoscope, and the writer decided that if he had not attended the course his slit-lamp would have been of little use to him. He had thought, and doubtless many others think, that the slit-lamp is simply a very superior instrument for focal illumination; it is this, but also very much more. Czapski's microscope used in combination with Gullstrand's slit-lamp opens an entirely new chapter in ophthalmology, an interesting but very difficult chapter.

The lecture on Tuesday was devoted to the four methods of illumination: observation in focal light; by transmitted light; by indirect lateral illumination; and in the mirror region. In the first case the object is seen in light directly thrown upon it, by light reflected from itself. In this wise we see the front of the cornea and the substance of the cornea, the iris, and the pupil margin; the capsules and the whole thickness of the lens. 
Transmitted light is light reflected from the iris and lens. Iris light enables us to see the cornea lighted up from behind, and lens light is useful to examine the pupillary margin of the iris. The direct light is so strongly reflected from the surface of the iris that the finer points may be lost in the dazzle, but are well seen by the softer reflected light. (The mirror light is obtained by closely approximating the slit-lamp to the microscope so as to make the angle between them as acute as possible.) The patient is niade io fix an object between the two so that the direction of his gaze bisects this angle. The observer now sees the image of the light and on the edge of the mirror so formed it is possible to observe the corneal endothelium as a delicate mosaic of cells, and the iens shagreen.

The practical work in the afternoon was devoted to mastering these methods of illumination and to practising deep localization with a narrow ribbon in the cornea, anterior chamber, lens and vitreous. When the students entered the dark room they found the slit-lamps dismantled and every adjustment thrown out. The first exercise was to reassemble the lamp and adjust it. I'igs' eyes were still used, first unaltered and then with the cornea removed for the examination of the lens. Artificial eyes were also employed to practise focussing the instruments and to obtain the mirror light.

We finished the second day still more impressed with the difficulty of the work.

Wednesdav's lecture was devoted to the cornea. The normal thickness, pathological thickening and thinning of the cornea were dealt with in turn. The professor spoke at some lengti upon his discovery that the corneal endothelium could be seen in the mirror area. The light is reflected from the inner aspect of the cells and these show up as yellowish hexagonal cells. The corneal nerves are easily detected as white branching lines in the illuminated prism of light formed by the slit-lamp. Folds in Descemet's membrane show up as lines which are white or dark according to how the light falls upon them.

In the afternoon the class began work upon patients who were chosen to demonstrate normal and pathological conditions in the cornea.

The lecture on Thursday began with the subject of the anterior chamber. Professor Vogt pointed out that with the slit-lamp it was almost always possible to demonstrate the existence of some sort of chamber in subjects, which, examined by the ordinary methods, appeared to have none. The existence of an "eddy", current in the anterior chamber caused by the difference in warmth between the cornea and the iris was mentioned. This can be seen with the slit-lamp only when the aqueous contains discrete particles 
such as cells. The detection of cells in the aqueous has a valuable clinical significance. They appear in sympathetic ophthalmia before any other sign, and if detected may indicate the removal of the exciting eye, and enable active treatment to be initiated several days earlier than would be the case were we depending upon orthodox methods of examination.

The most interesting and important part of this lecture dealt with the lens. Vogt was the first to point out that the slit-lamp showed layers of discontinuity in the lens, which, even in the youngest children, could always be demonstrated. He divided the lens into: capsule, a subcapsular dark area, then the cortex, the senile nucleus, and, finally, the embryonic nucleus. The anterior capsule seen in mirror light showed a shagreen, even in young children. This light also brought out the shagreen of the posterior capsule. The anterior capsule accurately focussed with the narrow ribbon appears as a fine bright line. Under it is a narrow dark line, and then we see the cortex as a cloudy band. Suitable adjustment of light brings out the anterior and posterior lens figures. Just behind the posterior capsule on the nasal side the remains of the hyaloid artery can be seen in the large majority of individuals.

In the afternoon patients were examined illustrating the subjects 8 of the lecture.

Friday was reserved for a lecture upon the iris.

The slit-lamp makes it easy to see that the stroma of the iris consists of two layers, the anterior and posterior mesodermic plates.

The anterior layer stops short at the "frill" (krause in German). Suitable lateral illumination 'will often demonstrate a definite cavity at the frill between the two layers, Fuchs' cleft. Changes in the iris due to senile alteration and to disease were considered in turn. The abnormalities of the pupil margin were mentioned and shown in the afternoon.

The lecture on Saturday was upon the vitreous. 'The normal structure of the vitreous and its changes in disease, such as detachment of the retina, myopia, and haemorrhage, were treated in turn. The slit-lamp shows that prolapse of the vitreous is far more common after such an operation as discission than we suppose. The micro-arc lamp is necessary for a complete examination of the vitreous. This apparatus, invented by Vogt, was demonstrated in the dark room in the afternoon.

There was a special lecture upon the sources of error in slitlamp work which was given in the afternoon.

In addition to the main course there were several interesting and valuable "side shows." 
On Thursday and Friday afternoons patients were provided for examination with red-free light. The apparatus consists of a micro-arc light housed in a suitable chamber. The carbons are fed by clockwork. The light is passed through a filter and emerges as a beam of green light. This is allowed to fall upon the temple of the patient and the fundus is observed with an ordinary reflecting ophthalmoscope with the mirror tilted at 45 degrees. The examination must be short for the light is very intense, and is harmful to the eye. For this reason it is not necessary to have an arc light with clockwork feed. The apparatus in use is made by Zeiss and costs about twenty-five pounds. A simpler lantern was made by Leitz, but appeaĩs to be no longer obtainable. The writer made inquiries and finds that Messrs. Koch, of Bahnhoff Strasse, Zürich, can supply the cheaper lantern for about seven pounds. With import duty it should be under ten pounds. Messrs. Zeiss also propose to bring out a simpler lantern at about fifteen pounds.

Red-free ophthalmoscopy is valuable in that small haemorrhages, invisible in white light, come out black and are easily detected. The yellow colouration of the macula is plainly seen, and the nerve fibres in the retina are visible. It is claimed that examination of the fundus with red-free light allows a diagnosis of tobacco amblyopia to be made forthwith from the characteristic changes seen at the macula.

Professor Vogt devoted part of his lecture on Saturday afternoon to this subject, which he illustrated by many beautiful drawings projected upon the screen with the epidiascope.

The professor gave a demonstration of a family of buphthalmic rabbits. Some time back at Basle he obtained a litter of three rabbits, one male and two females, all with buphthalmos. From these he has been able to breed as many as he wishes, all with buphthalmos. The question was considered in the light of Mendel's Law.

A demonstration was given in the basement of the hospital upon the professor's experiments in the production of cataract by infra-red rays of low refrangibility.

The light from an arc-lamp is first cooled by passage through a glass cell through which a current of water passes. It then traverses a trough filled with a solution of iodine in potassium iodide. The resulting red rays are concentrated upon a rabbit's eye by a double convex lens of rock-salt. The rabbit has been put to sleep by an injection of "Somnifen." In from one quarter to half an hour the lens becomes completely opaque. We were told that Messrs. Zeiss will attempt to produce a glass that will filter these red rays and prevent the formation of cataract, and it is hoped that spectacles will be made that will enable glass 
blowers to carry on their trade without the danger of cataract. It should be stated that some fifteen years ago a Committee of the Royal Society (the Glassworkers' Cataract Committee) took this research in hand. It was for this Committee that Sir William Crookes prepared synthetic glasses, possessing powers of selective absorption.

One of the most valuable demonstrations that was given by the professor referred to his method of taking bone-free $\mathrm{X}$ ray photographs of the eye. A small plate (use an oval dental plate) is pressed into the orbit on the inner aspect, and the tube is located at the side so that the rays fall upon the eye at an oblique angle. The exposure should be three seconds with a soft tube.

The resulting photograph shows the upper and lower lids, the cornea, and takes in about half the circumference of the eye. Inasmuch as the eye is photographed without the intervention of bone, almost any foreign body will show up. Lead-free glass, spicules of bone, or stone are plainly seen. A second exposure should be made with the plate pushed in above the eye. If a small pin be hooked into the conjunctiva, a fairly accurate localization is possible. We have now made this method a routine in our own practice, and have found it very useful. It is possible to get a photograph in fifteen minutes at a cost of sixpence. Small metallic fragments which are too small to show up in an ordinary exposure through bone are quite plain in a bone-free picture, and it is these small fragments, which have not sufficient kinetic energy to pass deeply into the eye, that can be demonstrated in the anterior segment. In two of our cases the fragment lay in the vitreous just behind the ciliary body.

Professor Henker demonstrated his apparatus for estimating the refraction of an eye by direct inspection with the small Gullstrand ophthalmoscope. Several members of the class were interested and some had thoughts of buying the apparatus. We asked Professor Henker to work out the refraction of some of the members. Unfortunately, the errors were so great, and the blinding effect of the investigation so great, that -we decided that the apparatus was a "wash-out."

Professor Hess gave a most lucid and interesting lecture demonstrating his apparatus for mapping out the loss of function in paresis of ocular muscles. This is so very practical that no ophthalmic hospital should be without it.

Professor Hess, formerly an ophthalmic surgeon, is now Professor of Physiology in the University of Zürich.

A black sheet of cloth, about two feet by one and a half, hangs upon a frame. On it are nine red dots equally spaced in two rows at each side and one down the middle. The patient holds a stick at the end of which is a green arrow. He has a green glass before 
one eye, and a red before the other. Thus he sees the red spots with one eye, and the green arrow with the other. He is asked to place the arrow upon the dots in turn. On his non-paralysed side he succeeds. He falls short of the dots in the middle line, and far short of the lateral dots. The figure formed by joining up the various positions is now measured up and transferred to a chart. The nature and position of the figure enables the muscle involved to be read off at a glance. The spectacles are now noversed and the patient fixes with the paralysed eye. The resulting chart in addition to giving the secondary deviation gives the amount of permanent contracture. A study of these two charts and especially of the contracture is of great value if an operation is proposed. The apparatus can be obtained from the Physiological Institute, Zürich.

We took the opportunity to accompany Professor Vogt round his wards. He starts at 7.45 and sees every patient daily. We thought that many of the patients would have been treated with us as out-patients, but the provision of ample beds enables such to have the advantage of in-patient treatment. Ophthalmia neonatorum is treated by injections of milk. The milk is boiled for a minute, and a second injection is made before the reaction of the first has passed off. Professor Vogt says that this method shortens the period of treatment very considerably. Serpent ulcer is treated by removal of diseased sac, by evacuation of the hypopyon by a peripheral corneal incision, and, finally, by the use of a "Priessnitz," which is really a fomentation of zinc sulphate in half per cent: solution. The professor stated that this treatment was most satisfactory. Blepharitis is handled with a powder containing zinc oxide and naftalan.* Buphthalmos is never left to nature. Either an iridencleisis is performed or repeated sclerotomies are done.

In the out-patient department refraction is carried out chiefly by estimation with the refracting ophthalmoscope, and with the ophthalmometer; retinoscopy is not often employed.

The notes, both in- and out-patient, ate kept in books, each of which has a separate index; there is no card index. We gathered that on an average twenty-five new patients attended daily, and the staff consists of the professor, four resident assistants, and occasional help from doctors working in the clinic. This allows five new patients a day to each, so perhaps the book system can be managed! The large amount of help and the comparatively small number of patients explains why it is possible to carry out the minute examination and the researches that we associate with continental work.

*Naftalan, 5.0 grammes; zinc oxide, 2.5 grammes: wheat starch, 2.5 grammes Especially recommended for blepharo-conjunctivitis with much secretion. 

3 p.m.

The professor sees his private patients at the clinic from 2 to

The whole course was most inspiring from every point of view. The lectures were copiously illustrated, chiefly from Vogt's Atlas of slit-lamp microscopy, and the instructors were untiring in their efforts to teach the difficult technique of the slit-lamp. We feel certain that no one is at all likely to get much value out of his slit-lamp unless he has attended a course of this nature. When one has mastered the technique, and what is more important has been shown what one ought to see, then practice alone is necessary to attain efficiency.

The course ended with a dinner on Saturday evening attended by the Rector of the University, Professor Vogt, Professor Hess, the Instructors, the Members of the Class, and others.

On our journey home we stopped at Basle and visited the eye clinic there. We had an opportunity to examine Nagel's Anomaloscope, an apparatus for testing colour perception by the Rayleigh Equation. The field of a telescope is divided by a horizontal line. The lower half is illuminated by sodium light. The upper half can be illuminated by red or green lights by turning a screw. The two lights are mixed till the upper and lower fields match. The proportion of red and green employed is read off on a scale, and from this the colour error can be deduced.

We were indebted to the first assistant, Dr. Koby, for showing us over the clinic. Professor Hallauer had not taken over his duties.

A large number of the beds in the clinic are allotted to private patients, who are divided into three classes. The fees for the first class amounted to five or six pounds a week, and about fifty guineas would be paid for an extraction. The second and third class pay less. The cost of the free patients is to a large extent defrayed by the various sick clubs and insurance schemes (Kranken Kassen).

Here again we were surpised to find that all the notes were taken in books, and we noted that both here and at Zürich the waiting room would accommodate only about forty patients, and in neither case did we ever see a patient waiting. 